\title{
Berberine Activates GLUT1-Mediated Glucose Uptake in 3T3-L1 Adipocytes
}

\author{
So Hui KIm, ${ }^{a}$ Eun-Jung Shin, ${ }^{a}$ Eun-Do KIM,${ }^{a}$ Tsenguun BayaraA, ${ }^{a}$ Susan Cooke Frost,${ }^{b}$ and \\ Chang-Kee HYuN*,a \\ ${ }^{a}$ School of Life and Food Sciences, Handong Global University; Pohang, Kyungbuk 791-708, Korea: and ${ }^{b}$ Department of \\ Biochemistry and Molecular Biology, University of Florida College of Medicine; Gainesville, FL 32606, U.S.A. \\ Received June 14, 2007; accepted August 25, 2007
}

It has recently been known that berberine, an alkaloid of medicinal plants, has anti-hyperglycemic effects. To explore the mechanism underlying this effect, we used 3T3-L1 adipocytes for analyzing the signaling pathways that contribute to glucose transport. Treatment of berberine to 3T3-L1 adipocytes for $6 \mathrm{~h}$ enhanced basal glucose uptake both in normal and in insulin-resistant state, but the insulin-stimulated glucose uptake was not augmented significantly. Inhibition of phosphatidylinositol 3-kinase (PI 3-K) by wortmannin did not affect the berberine effect on basal glucose uptake. Berberine did not augment tyrosine phosphorylation of insulin receptor (IR) and insulin receptor substrate (IRS)-1. Further, berberine had no effect on the activity of the insulinsensitive downstream kinase, atypical protein kinase $C(\mathrm{PKC} \zeta / \lambda)$. However, interestingly, extracellular signalregulated kinases (ERKs), which have been known to be responsible for the expression of glucose transporter (GLUT)1, were significantly activated in berberine-treated 3T3-L1 cells. As expected, the level of GLUT1 protein was increased both in normal and insulin-resistant cells in response to berberine. But berberine affected the expression of GLUT4 neither in normal nor in insulin-resistant cells. In addition, berberine treatment increased AMP-activated protein kinase (AMPK) activity in 3T3-L1 cells, which has been reported to be associated with GLUT1-mediated glucose uptake. Together, we concluded that berberine increases glucose transport activity of 3T3-L1 adipocytes by enhancing GLUT1 expression and also stimulates the GLUT1-mediated glucose uptake by activating GLUT1, a result of AMPK stimulation.

Key words berberine; glucose uptake; 3T3-L1 adipocytes; glucose transporter 1; extracellular signal-regulated kinase; AMPactivated protein kinase

Glucose uptake in insulin-sensitive tissues, which is dependent on the activity of glucose transporters (GLUTs), is a major regulatory process in the homeostatic control of blood glucose levels. GLUT1 is ubiquitously expressed for fulfilling basal glucose uptake, whereas GLUT4 is expressed in the insulin sensitive tissues in which glucose uptake is acutely stimulated by insulin. ${ }^{1)}$ The regulatory mechanisms for the activity of glucose transporters have been extensively studied. It has been known that, at least two distinct signaling pathways are implicated in insulin-stimulated GLUT4 translocation and glucose uptake. One pathway results from the tyrosine phosphorylation of insulin receptor substrate (IRS) protein, leading to the activation of phosphatidylinositol 3-kinase (PI 3-K), and a second pathway occurs through the tyrosine phosphorylation of $\mathrm{Clb}$, followed by the activation of TC10. ${ }^{2}$ Recent evidence has demonstrated that atypical protein kinase $\mathrm{C}(\mathrm{PKC} \zeta / \lambda)$ is known to be a convergent target of the insulin-stimulated PI $3-\mathrm{K}$ and TC10 pathways. ${ }^{3)}$ Related to GLUT1, it has been reported that activation of extracellular signal-regulated kinase (ERK) up-regulates GLUT1 expression, resulting in augmentation of basal glucose uptake in the absence of acute insulin stimulation. ${ }^{4,5)}$ In addition, an enhancement of GLUT1-mediated glucose transport is reportedly associated with an stimulation of AMP-activated protein kinase (AMPK). ${ }^{6,7)}$

Berberine, an isoquinoline alkaloid isolated from some Chinese medicinal herbs such as Coptidis Rhizoma and Cortex Phellodendri, has been used for the treatment of diarrhea an other gastrointestinal infections as an antibacterial drug in Chinese medicine. ${ }^{8)}$ Recently, it has been reported that berberine reduces serum cholesterol, triglycerides, and LDL- cholesterol in hypercholesterolemic patients, with being suggested as a novel cholesterol-lowing drug. ${ }^{9}$ Recent studies have also shown that berberine has antihyperglycemic effects. $^{10-12)}$ However, elucidations of berberine action are not in agreement between research groups. One suggested an insulin-independent effect, ${ }^{10)}$ whereas others showed an insulin-secretion stimulating ${ }^{11)}$ or insulin-sensitizing effects. ${ }^{13)}$ Although the mechanism underlying the berberine effect is not known in detail, it might involve the activation of AMPK. Lee et al. suggested that berberine has beneficial effects in diabetes and obesity in part via activation of AMPK. ${ }^{14)}$

Presently, we found that berberine displays an enhancing effect on GLUT1-mediated glucose transport in 3T3-L1 adipocytes. To explore a yet unidentified mechanism, we analyzed related signal transduction pathways that are activated by berberine.

\section{MATERIALS AND METHODS}

Materials Antibodies against IR, IRS-1, phospho-IRS-1 (Ser636/639), PKC $\zeta / \lambda$, phospho-PKC $\zeta / \lambda$ (Thr410/403), p38, phospho-p38 (Thr180/Tyr182), ERK1/2, phospho-ERK1/2 (Thr202/Tyr204), AMPK, and phospho-AMPK (Thr172) were purchased from Cell Signaling Technology (Beverly, MA, U.S.A.). Anti-GLUT1 and anti-GLUT4 antibodies were purchased from Santa Cruz Biotechnology, Inc. (Santa Cruz, CA, U.S.A.). Anti-phosphotyrosine antibody was purchased from Upstate Cell Signalling Solutions (Charlottesville, VA, U.S.A.). Unless specifically mentioned, reagents including berberine were purchased from Sigma-Aldrich (St. Louis, 
MO, U.S.A.).

Cell Culture and Induction of Insulin Resistance 3T3L1 fibroblasts were grown and differentiated as previously described by Frost and Lane. ${ }^{15)}$ To induce insulin resistance, cells were chronically exposed to insulin $(10 \mathrm{~nm})$ for $12 \mathrm{~h}$ in the presence or absence of berberine. Before the glucose transport assay or in the subcellular fractionation studies, cells were washed 3 times with $3.0 \mathrm{ml}$ Krebs Ringer phosphate buffer (KRP) containing glucose $(5 \mathrm{~mm})$ and bovine serum albumin (BSA; $1 \mathrm{~g} / \mathrm{l})$ at 40-min intervals over a 2 -h period. 3T3-L1 adipocytes were treated or not with berberine at specific concentrations $(1-10 \mu \mathrm{M})$ for the times noted in the figure legends. DMSO was used as vehicle for untreated cells.

Glucose Transport Assay Cytochalasin B-inhibitable glucose transport was assayed as previously described by Thomson et al. ${ }^{16)}$ with some modifications. Briefly, 3T3-L1 adipocytes were exposed to berberine for the times indicated in complete DMEM containing 10\% FBS. Cell monolayers were rapidly washed in KRP at $\mathrm{pH} 7.4$ and incubated with or without $20 \mu \mathrm{M}$ cytochalasin $\mathrm{B}$ for $10 \mathrm{~min}$ at $37^{\circ} \mathrm{C}$. Insulin $(100 \mathrm{~nm})$ was added or not for $10 \mathrm{~min}$, and then $\left[{ }^{3} \mathrm{H}\right] 2$ deoxyglucose $(0.2 \mathrm{~mm}, 0.2 \mu \mathrm{Ci})$ was added for an additional $10 \mathrm{~min}$. Glucose uptake was terminated with 3 washes of icecold PBS. Cells were dissolved in $0.1 \%$ sodium dodecyl sulfate (SDS) and counted for radioactivity.

Immunoprecipitation of IR and IRS-1 Immunoprecipitation of IR and IRS-1 was conducted as previously described by Hwang and Frost ${ }^{17)}$ with some modifications. Proteins released from Protein A Sepharose beads were separated by $7.5 \%$ SDS-PAGE followed by transfer to nitrocellulose. Blots were screened for total and tyrosine-phosphorylated protein.

Analysis of PKC $\zeta / \lambda$, p38, ERK1/2 and AMPK To test for the involvement of $\mathrm{PKC} \zeta / \lambda, \mathrm{p} 38, \mathrm{ERK} 1 / 2$ and AMPK, cells were extracted in lysis buffer $(140 \mathrm{~mm} \mathrm{NaCl}, 20 \mathrm{~mm}$ Tris/ $\mathrm{HCl} \mathrm{pH}$ 7.5, $1 \mathrm{~mm}$ EDTA, $100 \mathrm{~mm} \mathrm{NaF,} 10 \mathrm{~mm} \mathrm{Na} \mathrm{PO}_{4}$, $1 \mathrm{~mm} \mathrm{Na} \mathrm{VO}_{4}, 1 \mathrm{~mm}$ phenylmethylsulfonyl fluoride, $1 \% \mathrm{NP}-$ 40 in the presence of protease inhibitors). The extract was tumbled for $30 \mathrm{~min}$ at $4{ }^{\circ} \mathrm{C}$ and clarified by spinning at $16000 \mathrm{~g}$ at $4{ }^{\circ} \mathrm{C}$ for $10 \mathrm{~min}$. Proteins in the clarified supernatant were separated by SDS-PAGE and transferred to nitrocellulose. The concentration of total each protein was compared against the phosphorylated pool by Western blot analysis.

Membrane Isolation Total membranes were isolated from cells as described by Kitzman et al. ${ }^{18)}$ Proteins were separated by SDS-PAGE and transferred to nitrocellulose for Western blot analysis.

Western Blot Analysis For IR, IRS-1, phospho-IRS-1, $\mathrm{PKC} \zeta / \lambda$, phospho-PKC $\zeta / \lambda$, p38, phospho-p38, ERK1/2, phospho-ERK1/2, AMPK, phospho-AMPK, GLUT1 and GLUT4, the Western blotting procedure was described previously ${ }^{16,17)}$ with some modifications. Briefly, cells were rinsed with ice-cold PBS and solubilized in a lysis buffer containing $50 \mathrm{~mm}$ HEPES, $150 \mathrm{~mm} \mathrm{NaCl}, 10 \mathrm{~mm}$ EDTA, 0.5\% Triton X$100,5 \%$ glycerol, $0.25 \%$ Nonidet P-40, 1 mm phenylmethylsulphonyl fluoride (PMSF), $1 \mu \mathrm{g} \mathrm{ml}^{-1}$ aprotinin, $1 \mu \mathrm{g} \mathrm{ml}^{-1}$ leupeptin, $100 \mathrm{~mm} \mathrm{NaF}$ and $1 \mathrm{~mm} \mathrm{Na}_{3} \mathrm{VO}_{4}$ (pH 7.4) and incubated for $30 \mathrm{~min}$ at $4^{\circ} \mathrm{C}$. The whole-cell lysates were centrifuged at $4{ }^{\circ} \mathrm{C}$ for $10 \mathrm{~min}$ to remove the insoluble materials.
The proteins in the supernatants were separated by $10 \%$ SDS-PAGE and transferred to nitrocellulose membranes. After blocking with TBS-T $(10 \mathrm{~mm}$ Tris, $150 \mathrm{~mm} \mathrm{NaCl}$, $0.05 \%$ Tween-20, pH 7.6) containing 5\% nonfat milk for $1 \mathrm{~h}$ at room temperature, the membranes were incubated with the appropriate antibodies. The proteins were visualized by enhanced chemiluminescence using horseradish peroxidaseconjugated anti-rabbit or mouse IgG. Densitometric analysis of the bands was performed using AlphaImager 2200 (Alpha Innotech Inc., San Leandro, CA, U.S.A.) and analyzed by AlphaEase FC software (Alpha Innotech Inc.).

Statistical Analysis Numerical data are reported as means \pm S.E.M. with the number of replicates indicated in the figure legends. Each graphical point represents an independent data set whether assayed for time or treatment. Data were analyzed by the ANOVA procedure of Statistical Analysis System (SAS Institute, 1999-2001). Significant differences between groups were determined using Duncan's multiple range tests at the $p<0.05$ level.

\section{RESULTS}

Berberine Enhances Basal Glucose Uptake Both in Normal and Insulin-Resistant 3T3-L1 Adipocytes Incubation of 3T3-L1 adipocytes with berberine enhanced glucose transport activities significantly both in normal and insulin-resistant state (Fig. 1). In normal cells, exposure to $5 \mu \mathrm{M}$ berberine for $6 \mathrm{~h}$ resulted in approximately 8.5 -fold increase in insulin-independent (basal) glucose uptake and 1.3-fold in insulin-stimulated glucose uptake. Chronic exposure to $10 \mathrm{~nm}$ insulin for $12 \mathrm{~h}$ reduced insulin-stimulated glucose uptake by $50 \%$, which was defined as an insulin-resistant state. Even though glucose uptake in insulin-resistant cells was also augmented, no significant additive response was observed on berberine-induced glucose uptake. Timeand dose-dependent experiment showed that stimulation of $\left[{ }^{3} \mathrm{H}\right] 2$-deoxyglucose uptake was maximal at a 6 -h incubation period with $5 \mu \mathrm{M}$ berberine (data-not-shown), which condition was employed in subsequent experiments to test the effect of berberine.

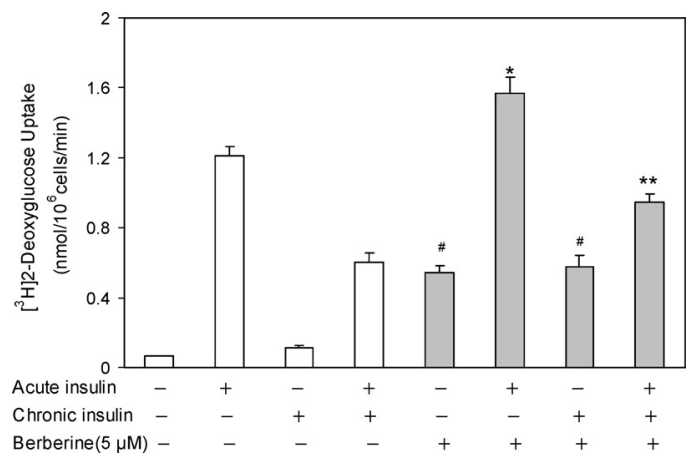

Fig. 1. Berberine Enhances Glucose Transport in Normal and InsulinResistant 3T3-L1 Adipocytes

Cells were exposed to $5 \mu \mathrm{M}$ berberine for $6 \mathrm{~h}$ in the absence or presence of chronic exposure to $10 \mathrm{nM}$ insulin for $12 \mathrm{~h}$, then washed three times in KRP containing $0.5 \%$ $\mathrm{BSA}$ at $40 \mathrm{~min}$-intervals over $2 \mathrm{~h}$ at $37^{\circ} \mathrm{C}$. Insuin $(100 \mathrm{~nm})$ was added or not for $10 \mathrm{~min}$. [ ${ }^{3} \mathrm{H}$ ]2-Deoxyglucose $(0.2 \mathrm{~mm}, 0.2 \mu \mathrm{Ci})$ was added for an additional $10 \mathrm{~min}$. Transport activity was terminated with 3 washes of ice-cold PBS. Cells were lysed in $0.1 \%$ SDS and counted for radioactivity. Data represent duplicate experiments of cytochalasin Binhibitable uptake. $\# p<0.01$ compared to basal value of normal cells, $* p<0.05 \mathrm{com}$ pared to insulin-stimulated value of normal cells, $* * p<0.05$ compared to insulinstimulated value of insulin-resistant cells. 


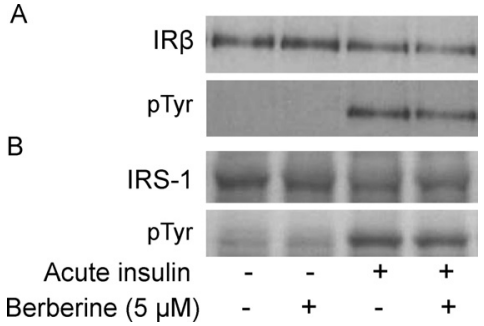

Fig. 2. Berberine Does Not Affect the Receptor Number or Autophosphorylation of IR, nor the Pool or Phosphorylation of IRS-1

Cells were washed extensively and acutely exposed or not to $100 \mathrm{~nm}$ insulin for $10 \mathrm{~min}$. After washing, cells were scraped in a buffer and a total membrane fraction was isolated. After extraction, the lysates was immunoprecipitated with anti-IR (A), antiIRS-1 (B) followed by immunoblot analysis with anti-phosphotyrosine antibodies as indicated. Representative immunoblots of 3 independent experiments are shown.

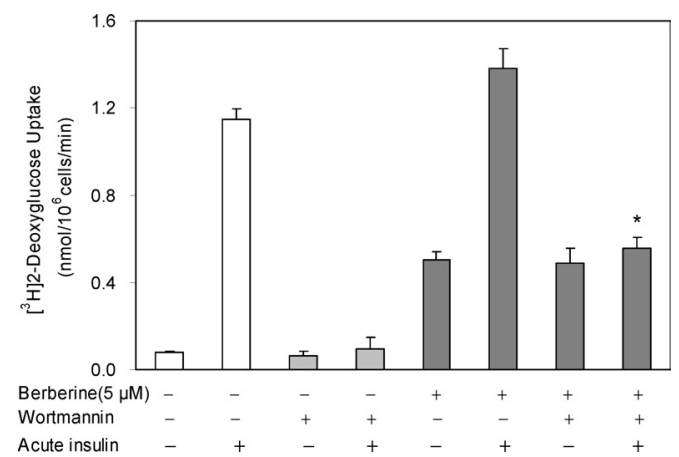

Fig. 3. Wortmannin Does Not Block Berberine-Induced Glucose Uptake

Cells were exposed to $5 \mu \mathrm{m}$ berberine for $6 \mathrm{~h}$, then rinsed in KRP and incubated with or without $100 \mathrm{~nm}$ wortmannin for $10 \mathrm{~min}$. Insuin $(100 \mathrm{nM})$ was added or not for an additional $10 \mathrm{~min}$ before deoxyglucose uptake was assessed. Data represent duplicate experiments of cytochalasin B-inhibitable uptake. $* p<0.01$ compared to insulin-stimulated value of berberine-treated cells.

Berberine Dose Not Affect Insulin-Responsive Signal Transduction Pathways To explore the effect of berberine on glucose transport activity, we first looked at an event upstream of PI 3-kinase activation, i.e., IR autophosphorylation and IRS-1 phosphorylation. However, any significant change was observed neither in the receptor pool or catalytic activity in response to berberine treatment (Fig. 2). Also in the case of protein pool or tyrosine phosphorylation of IRS1 , berberine had no effect.

We next utilized the PI 3-kinase inhibitor, wortmannin, to determine if the PI 3-kinase pathway is involved in berberine-induced sensitization,. As shown by others, ${ }^{19,20)}$ insulinsensitive glucose uptake is completely blocked by wortmannin (Fig. 3). In berberine-treated cells, wortmannin was clearly inhibitory, but the inhibition was not as complete as in the control cells. It was observed that the enhancement of basal glucose uptake by berberine was remained not to be blocked. This suggests that there may be PI 3-kinase independent path by which berberine enhances glucose transport activity.

We next focused on $\mathrm{PKC} \zeta / \lambda$ phosphorylation. Insulin stimulation results in the phosphorylation of $\operatorname{PKC} \zeta / \lambda$, which is known to be a convergent target of the insulin-stimulated PI 3-K and TC10 pathways, ${ }^{3)}$ to mediate insulin-induced increases in glucose transport. As expected, acute insulin exposure stimulated $\mathrm{PKC} \zeta / \lambda$ phosphorylation at $\mathrm{Thr} 410 / 403$ without affecting the total pool (Fig. 4). However, berberine treatment had no effect on the extent of phosphorylation.

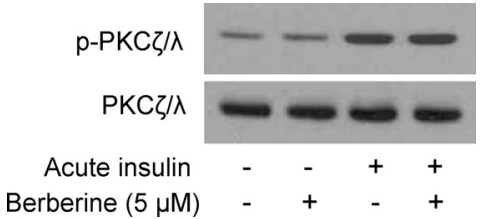

Fig. 4. Berberine Does Not Affect Total Pool or Phosphorylation of $\operatorname{PKC} \zeta / \lambda$

Cells were treated with berberine for $6 \mathrm{~h}$. Cells were rinsed in KRP and acutely exposed or not to $100 \mathrm{~nm}$ insulin for $10 \mathrm{~min}$. After washing and lysis, total cell lysates was analyzed by immunoblot to measure total $\mathrm{PKC} \zeta / \lambda$ and phospho-PKC $\zeta / \lambda$. Representative immunoblots of 3 independent experiments are shown.

\section{A}

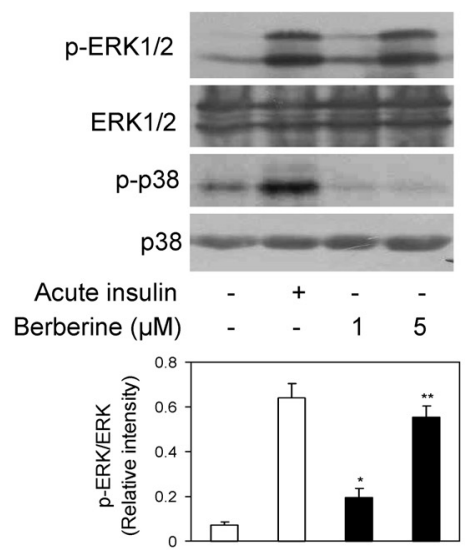

B

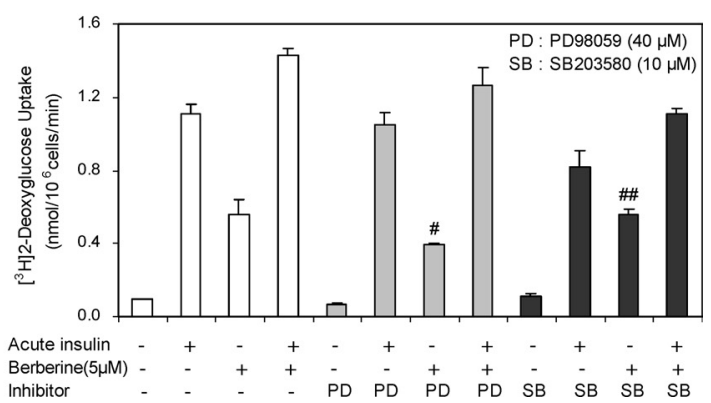

Fig. 5. Berberine Induces Phosphorylation of ERK, But Not of p38

(A) Cells were treated with berberine $(1$ or $5 \mu \mathrm{M})$ for $6 \mathrm{~h}$ or insulin $(100 \mathrm{nM})$ for $10 \mathrm{~min}$. After washing and lysis, total cell lysates was analyzed by immunoblot to measure total ERK, phospho-ERK, p38, and phospho-p38. Data are expressed as the mean value \pm S.E.M. of 3 independent experiments. $* p<0.05$, $* * p<0.01$ compared to relative intensity value of cells neither exposed to insulin nor to berberine. (B) Cells were exposed to $5 \mu \mathrm{M}$ berberine for $6 \mathrm{~h}$, then rinsed in KRP and incubated with or without $40 \mu \mathrm{M}$ PD98059 or $10 \mu \mathrm{M}$ SB203580 for $30 \mathrm{~min}$. Insulin $(100 \mathrm{nM})$ was added or not for an additional $10 \mathrm{~min}$ before deoxyglucose uptake was assessed. Data represent duplicate experiments of cytochalasin B-inhibitable uptake. $\# p<0.05$ compared to basal glucose transport value of berberine-treated cells, \#\#n significance.

Berberine Induces Activation of ERK and AMPK Next, we examined the effect of berberine on acute, insulinstimulated MAPK phosphorylation. It has been known that the activation of MAPK kinase $1 / 2$ (MKK1/2)-ERK or MKK6/3-p38 pathway leads to upregulation of GLUT1 expression which in turn enhances basal glucose uptake in 3T3L1 adipocytes. ${ }^{5)}$ As shown in Fig. 5A, berberine treatment resulted in a significant increase in ERK phosphorylation as determined by immunoblot of phospho-ERK1/2 without changing the total amount of ERK. In contrast, berberine had no effect on p38 activation. Both of total pool and phospho- 
rylation of p38 were unaffected by berberine treatment. The consistent results were obtained when PD98059 and SB203580, specific inhibitors of ERK and p38, respectively, were employed in glucose transport assay. Inhibition of ERK by PD98059 caused a significant decrease in berberine-stimulated glucose uptake, but treatment with SB203580 did not make any change (Fig. 5B).

To further examine the effect of berberine on GLUT1mediated glucose transport, we analyzed phosphorylation of AMPK by western blotting. It has recently been reported that signal pathway of GLUT1-mediated glucose transport involves AMPK stimulation. ${ }^{6,7)}$ As shown in Fig. 6A, a significant increase of phosphorylated AMPK could be detected in response to berberine treatment. It was similar effect with that of exposure to 5-aminoimidazole-4-carboxamide riboside (AICAR), an AMPK agonist. Consistently, co-treatment with berberine and AICAR caused an additive increase in

A

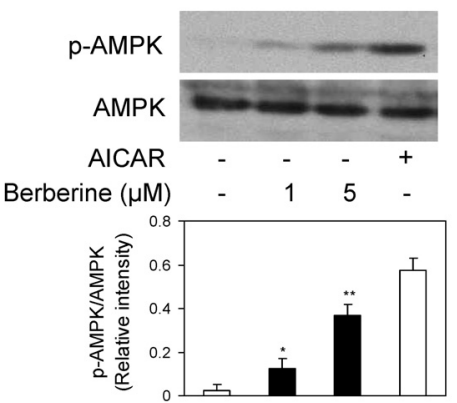

B

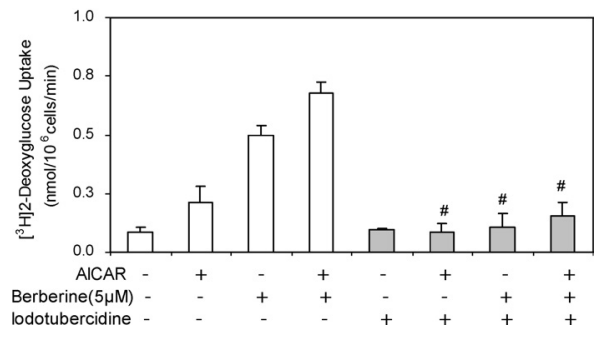

Fig. 6. Berberine Activates AMPK

(A) Cells were treated with berberine $(1$ or $5 \mu \mathrm{M})$ for $6 \mathrm{~h}$ or AICAR $(1 \mathrm{~mm})$ for $1 \mathrm{~h}$. After washing and lysis, total cell lysates was analyzed by immunoblot to measure total AMPK and phospho-AMPK. Data are expressed as the mean value \pm S.E.M. of 3 independent experiments. $* p<0.05, * * p<0.01$ compared to relative intensity value of cells neither exposed to AICAR nor to berberine. (B) Cells were pretreated with $10 \mu \mathrm{M} 5$ iodotubercidine or not for $10 \mathrm{~min}$, followed by exposure to $0.5 \mathrm{~mm}$ AICAR for $1 \mathrm{~h}$ or $5 \mu \mathrm{M}$ berberine for $6 \mathrm{~h}$ just before assessing deoxyglucose uptake. Data represent duplicate experiments of cytochalasin B-inhibitable uptake. $\# p<0.01$ compared to basal glucose transport values of cells without pretreatment of iodotubercidine. glucose uptake (Fig. 6B). In addition, using 5-iodotubercidine, a specific inhibitor of adenosine kinase, it was found that both of AICAR- and berberine-stimulated glucose uptake were nearly completely blocked by exposure to 5iodotubercidine.

Berberine Increases Expression of GLUT1 in 3T3-L1 Adipocytes To determine the effect of berberine on GLUT1 and GLUT4 expression, we analyzed their contents in total membranes by western blotting. As expected, the amount of GLUT1 was increased significantly in berberinetreated cells (Fig. 7). In contrast, the level of GLUT4 remained constant even after berberine treatment, showing that berberine had no effect on GLUT4 expression. To better understand the effect of berberine, expression levels of GLUT1 and GLUT4 were examined also in insulin-resistant cells. In agreement with glucose transport data (Fig. 1), expression of GLUT1 which is responsible for the enhancement of insulin-independent glucose uptake was increased also in insulin-resistant cells (Fig. 7). In the case of GLUT4, chronic exposure to insulin decreased the biosynthesis of GLUT4, but additional treatment with berberine did not change the GLUT4 level in insulin-resistant state.

\section{DISCUSSION}

Our data in this study show that, in 3T3-L1 adipocytes, insulin-independent (basal) glucose uptake is significantly increased in response to berberine. While Ko et $a l .{ }^{13)}$ reported that berberine doesn't stimulate glucose uptake without insulin, berberine alone could enhance glucose uptake in our study. In addition, the extent of increase in insulin-stimulated glucose uptake did not exceed that of basal glucose uptake in berberine-exposed cells, suggesting that there were no additive or synergistic effects between berberine and insulin. Hence, it was indicated that berberine has 'insulinlike' rather than 'insulin-sensitizing' effect. However, berberine did not block the development of insulin resistance induced by chronic exposure of low concentration $(12 \mathrm{~h}$, $10 \mathrm{~nm}$ ) of insulin.

To explore the mechanism underlying the effects of berberine, we examined several steps in the insulin-signaling cascade. First, an increase in the receptor pool or in autophosphorylation in response to berberine can be expected on the consumption that berberine might have an 'insulinlike' activity, but berberine did not alter catalytic activity of IR. Next, we examined the effect of berberine on tyrosine phosphorylation of IRS-1 in assessing the specific involvement of the PI 3-kinase pathway. It has been suggested that insulin-induced translocation of GLUT4 occurs by both PI 3-
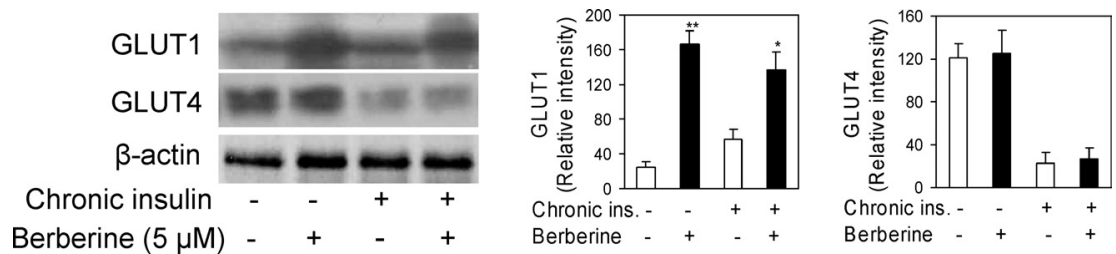

Fig. 7. Berberine Increases Expression of GLUT1, But Does Not Affect GLUT4 Pool

Cells were exposed to $5 \mu \mathrm{M}$ berberine for $6 \mathrm{~h}$ with or without chronic exposure to $10 \mathrm{~nm}$ insulin for $12 \mathrm{~h}$, then washed three times in KRP containing $0.5 \%$ BSA at 40 min-intervals over $2 \mathrm{~h}$ at $37^{\circ} \mathrm{C}$. Cells were scraped in a buffer and total membranes were isolated. Total protein levels were analyzed by immunoblot to measure GLUT1 and GLUT4. Data are expressed as the mean value \pm S.E.M. of 3 independent experiments. $* * p<0.01, * p<0.05$ compared to relative intensity value of berberine-untreated cells in normal and insulin-resistant state, respectively. 
kinase-dependent (IRS-1-PI 3-kinase) and independent (cCbl-TC10) paths. ${ }^{2)}$ Also in this step, however, berberine did not make any change. Like enhancing effect of berberine on glucose transport activity in 3T3-L1 adipocytes, this result was different from that of Ko et al., ${ }^{13)}$ in which berberine stimulated IRS-1 phosphorylation. Further, although wortmannin was used as a specific inhibitor of PI 3-kinase, it did not block the increase in glucose uptake stimulated by berberine treatment, suggesting that berberine may not augment signaling events downstream of PI 3-kinase. Next possibility that GLUT4 translocation might be stimulated by berberine is via TC10 pathway. Activation of PI 3-kinase by insulin stimulation leads to the activation of a downstream enzyme $\operatorname{PKC} \zeta / \lambda,{ }^{21)}$ which is also a target of TC10 pathway. ${ }^{3)}$ Also in our hands, acute addition of insulin stimulated $\operatorname{PKC} \zeta / \lambda$ phosphorylation. However, berberine did not affect the activation of $\mathrm{PKC} \zeta / \lambda$, indicating finally that the berberine effect is not related to PI 3-kinase nor TC10 pathways. Although the recent study by Lee and colleagues ${ }^{14)}$ showed that berberine stimulates GLUT4 translocation in L6 myotubes, it seems unlikely that the same process occur in 3T3-L1 adipocytes.

The increased intrinsic activity of GLUT1 as well as GLUT4 could potentially induce the enhancement of glucose uptake in 3T3-L1 adipocytes. In addition, it has been reported that GLUT1 expression can be up-regulated by activation of ERK resulting in an enhanced glucose transport. ${ }^{5,22}$ Fingar and Birnbaum showed that an activated form of Raf-1 (an upstream stimulator of MKK1/2 and ERK) increased the cellular pool of GLUT1 by 5-fold in 3T3-L1 adipocytes. ${ }^{4)}$ Our data show that berberine increased ERK phosphorylation significantly and a specific inhibitor of ERK impaired berberine-stimulated glucose uptake. When these data are taken together with the fact of ERK involvement in GLUT1 up-regulation, it is indicated that berberine might up-regulate GLUT1 expression, thereby augmenting GLUT1-mediated glucose transport. Further, we confirmed that berberine had an enhancing effect on the biosynthetic pool of GLUT1 determined by immunoblot analysis. Thus, our results demonstrate that berberine enhances GLUT1 expression by stimulating ERK pathway. In contrast, p38 MAPK did not appear to be involved in berberine-stimulated glucose uptake. Although some studies implicate p38 in the regulation of GLUT1 expression, ${ }^{23}$ berberine showed no effect on $\mathrm{p} 38$ phosphorylation in present study.

On the other hand, it has been shown that chronic activation of ERK can lead to not only up-regulation of GLUT1 but also down regulation of GLUT4 expression, resulting in a decreased insulin-stimulated glucose uptake. ${ }^{22}$ However, in the case of berberine-activated glucose transport activity, the signaling events were found to be different. The amount of GLUT4 in total membranes was not affected by berberine treatment. Although the induction of insulin resistance by exposing cells to chronic insulin reduced GLUT4 expression significantly, the GLUT4 levels in berberine treated cells were unchanged in both of normal and insulin-resistant states. This suggests that berberine may augment ERK activation to enhance GLUT1 expression without causing a decreased expression of GLUT4.

AMPK plays a critical role in cellular energy homeostasis, stimulating pathways which increase energy production such as glucose transport and fatty acid oxidation in response to a change in ATP/AMP ratio. ${ }^{24)}$ Among several major insulinsensitizing agents, the thiazolidinediones (TZDs) and metformin are thought to have beneficial effects, at least in part, by activating AMPK. ${ }^{25-27)}$ Interestingly, berberine was recently reported to activate AMPK in adipocytes, myotubes, and liver. ${ }^{14)}$ Consistent with those findings, also in our hands, AMPK phosphorylation was increased significantly in 3T3L1 cells after treatment with berberine. Further, berberinestimulated glucose uptake was almost completely abolished by exposure to a specific inhibitor of AMPK, ensuring a direct involvement of AMPK in enhancing glucose transport by berberine. Several researchers have reported that stimulation of AMPK activity is associated with enhancement of GLUT1-mediated glucose transport. ${ }^{6,7,28)}$ The underlying mechanism shows that the enhanced response of glucose transport is mediated by activation of GLUT1 preexisting in the plasma membrane without affecting the cell-surface concentration of GLUT1. Collectively, the role of AMPK is critical in stimulation of glucose transport via GLUT1 activation and AMPK is involved in berberine-stimulated glucose transport, leading to a conclusion that berberine may augment the ability of GLUT1 to accelerate glucose transport by activating AMPK.

In summary and conclusion, berberine enhances insulinindependent glucose uptake, which is a result of increased GLUT1 expression via ERK pathway. In addition, berberine further stimulates the GLUT1-mediated glucose uptake by activating GLUT1 proteins in the plasma membrane via AMPK pathway. The enhancing effect on GLUT1-dependent glucose uptake without diminishing GLUT4 expression can be considered as one of the beneficial feature of berberine as a potential anti-diabetic agent.

Acknowledgment This work was supported by the Korea Research Foundation Grant funded by the Korean Government (MOEHRD, Basic Research Promotion Fund) (KRF-2006-521-F00079).

\section{REFERENCES}

1) Olson A. L., Pessin J. E., Annu. Rev. Nutr., 16, 235-256 (1996).

2) Bryant N. J., Govers R., James D. E., Nat. Rev. Mol. Cell Biol., 3, $267-277$ (2002).

3) Kanzaki M., Mora S., Hwang J. B., Saltiel A. R., Pessin J. E., J. Cell. Biol., 164, 279-290 (2004).

4) Fingar D. C., Birnbaum M. J., J. Biol. Chem., 269, 10127-10132 (1994).

5) Fujishiro M., Gotoh Y., Katagiri H., Sakoda H., Ogihara T., Anai M., Onishi Y., Ono H., Abe M., Shojima N., Fukushima Y., Kikuchi M., Oka Y., Asano T., Mol. Endocrinol., 17, 487-497 (2003).

6) Abbud W., Habinowski S., Zhang J. Z., Kendrew J., Elkairi F. S., Kemp B. E., Witters L. A., Ismail-Beigi F., Arch. Biochem. Biophys., 380, 347-352 (2000).

7) Barnes K., Ingram J. C., Porras O. H., Barros L. F., Hudson E. R., Fryer L. G. D., Foufelle F., Carling D., Hardie D. G., Baldwin S. A., J. Cell. Sci., 115, 2433-2442 (2002).

8) Ni Y. X., Zhong Xi Yi Jie He Za Zhi, 8, 711-713 (1988)

9) Kong W., Wei J., Abidi P., Lin M., Inaba S., Li C., Wang Y., Wang Z., Si S., Pan H., Wang S., Wu J., Wang Y., Li Z., Liu J., Jiang J. D., Nat. Med., 10, 1344-1351 (2004).

10) Yin J., Hu R., Chen M., Tang J., Li F., Yang Y., Chen J., Metabolism, 51, 1439-1443 (2002).

11) Leng S. H., Lu F. E., Xu L. J., Acta Pharmacol. Sin., 25, 496-502 (2004). 
12) Bian X., He L., Yang G., Bioorg. Med. Chem. Lett., 16, 1380-1383 (2006).

13) Ko B. S., Choi S. B., Park S. K., Jang J. S., Kim Y. E., Park S., Biol. Pharm. Bull., 28, 1431-1437 (2005).

14) Lee Y. S., Kim W. S., Kim K. H., Yoon M. J., Cho H. J., Shen Y., Ye J.M., Lee C. H., Oh W. K., Kim C. T., Hohnen-Behrens C., Gosby A., Kraegen E. W., James D. E., Kim J. B., Diabetes, 55, 2256-2264 (2006).

15) Frost S. C., Lane M. D., J. Biol. Chem., 260, 2646-2652 (1985).

16) Thomson M. J., Williams M. G., Frost S. C., J. Biol. Chem., 272, 7759-7764 (1997).

17) Hwang J. B., Frost S. C., J. Biol. Chem., 274, 22813-22820 (1999).

18) Kitzman H. H., McMahon R. J., Williams M. G., Frost S. C., J. Biol. Chem., 268, 1320-1325 (1993).

19) Clarke J. F., Young P. W., Yonezawa K., Kasuga M., Holman G. D., Biochem. J., 300, 631-635 (1994).

20) Okada T., Kawano Y., Sakakibara T., Hazeki O., Ui M., J. Biol. Chem., 269, 3568-3573 (1994).
21) Farese R. V., Am. J. Physiol. Endocrinol. Metab., 283, E1-E11 (2002).

22) Fujishiro M., Gotoh Y., Katagiri H., Sakoda H., Ogihara T., Anai M., Onishi Y., Ono H., Funaki M., Inukai K., Fukushima Y., Kikuchi M., Oka Y., Asano T., J. Biol. Chem., 276, 19800-19806 (2001).

23) Yamamoto Y., Yoshimasa Y., Koh M., Suga J., Masuzaki H., Ogawa Y., Hosoda K., Nishimura H., Watanabe Y., Inoue G., Nakao K., Diabetes, 49, 332-339 (2000).

24) Daval M., Foufelle F., Ferre P., J. Physiol., 574, 55-62 (2006).

25) Fryer L. G. D., Parbu-Patel A., Carling D., J. Biol. Chem., 277, 25226-25232 (2002).

26) Ye J. M., Dzamko N., Cleasby M. E., Hegarty B. D., Furler S. M., Cooney G. J., Kraegen E. W., Diabetologia, 47, 1306-1313 (2004).

27) Hawley S. A., Gadalla A. E., Olsen G. S., Hardie D. G., Diabetes, 51, $2420-2425$ (2002).

28) Jing M., Ismail-Beigi F., Am. J. Physiol. Cell Physiol., 290, 484- 491 (2006). 